\title{
Collisionless heating in capacitive discharges enhanced by dual frequency excitation
}

\author{
M. M. Turner* \\ School of Physical Sciences and National Centre for Plasma Science and Technology, \\ Dublin City University, Dublin 9, Ireland \\ P. Chabert \\ Laboratoire de Physique et Technologie des Plasmas, \\ Ecole Polytechnique, 91128 Palaiseau, France
}

(Dated: February 28, 2008)

\begin{abstract}
We discuss collisionless electron heating in capacitive discharges excited by a combination of two disparate frequencies. By developing an analytical model, we find, contrary to expectation, that the collisionless heating effect is much larger in the presence of two frequencies than when either frequency acts alone. This prediction is substantiated by kinetic simulations, which are also in excellent general quantitative agreement with the model for discharge parameters that are typical of recent experiments.
\end{abstract}


Interaction with an electrode is a central problem in the physics of confined plasmas, whether the focus be fundamentals or applications. An important instance of this problem occurs when the electrode carries an oscillating voltage. The sheath surrounding the electrode then becomes an oscillatory structure of great intrinsic interest and practical significance. Especially remarkable physical effects occur when particle collisions within the sheath are rare, because substantial amounts of power may nevertheless be dissipated in the vicinity of the electrode, and discharges may be sustained predominantly by this so-called collisionless heating process $[1,2]$. Recently, new experimental contexts have appeared, with the rise of interest in dual-frequency capacitive discharges [3-5], and the related need to widen the range of frequencies that are used, including much larger frequencies that have been usual. In this Letter, we investigate the collisionless heating mechanisms that act in such cases. For typical parameters, we find a surprising result, that the heating produced by the superposition of two currents with different frequencies can be much larger than the effect of either acting alone, contrary to recent predictions of an additive effect [5]. We also show that for typical experimental conditions, the theory can be reduced to a closed formula, suitable for use in simple analytic models.

Despite the evident importance of collisionless heating effects in capacitive discharge experiments [1] and simulations [6, 7], a consistent theory, or even a clear physical model [6-11], has proved elusive. Both Godyak [8] and Lieberman [9] emphasized physical models in which electrons are heated by collision with a moving sheath edge, localizing the heating mechanism at a point. If one takes these models seriously, one expects the heating effect to be localized in a transition region between the quasi-neutral plasma and the positive space charge sheath. However, simulations demonstrate that such a transition region plays little or no essential role [7]. Moreover, it can be shown that the models discussed by Godyak and Lieberman fail to conserve current through the sheath region, and that when these models are modified such that current conservation is enforced, the collisionless heating effect exactly vanishes. Gozadinos et al [6] both articulated and addressed these arguments, by developing an approach where the electron fluid in the sheath region is described by moment equations with a simple kinetic closure. This model exactly conserves current, explicitly takes into account the spatial structure of the sheath, contains no adjustable parameters, and is in good agreement with kinetic simulation results. Kaganovich [11] has since developed a kinetic treatment in which current is conserved. This however represents 
the ion density in the sheath as a step function, and introduces the main collisionless heating effect via an ansätz. Moreover, this model is in less good agreement with the simulation results given by Gozadinos et al. Therefore, we regard the moment model discussed in [6] as the most satisfactory of the available theories, and it is the point of embarkation for the present discussion.

We consider a collisionless plasma consisting of electrons and one species of positive ion. An electrode in contact with the plasma carries a net current density given by $J(t)=$ $-\tilde{J}_{l} \sin \omega_{l} t-\tilde{J}_{h} \sin \omega_{h} t$, where $\tilde{J}_{l, h}$ and $\omega_{l, h}$ are distince current density amplitudes and angular frequencies. The structure of the boundary region that forms adjacent to the electrode is discussed elsewhere $[4,12]$. Briefly, it is useful to identify an electron sheath edge, defined as a point separating the positive space charge sheath from the quasi-neutral plasma. The electron sheath edge traces a complex path as the phase of the current advances [4], but this motion is bounded, and we define as the ion sheath edge the point where the electron sheath edge is most distant from the electrode. The ion density is then uniform on the plasma side of the ion sheath edge, and rather rapidly diminishing on the electrode side, as the ions are accelerated towards the electrode. We consider that the collisionless heating effect occurs in the electron fluid, in the region between the electron and ion sheath edges. This region is described by the Vlasov-Poisson system of equations. Using moments of the Vlasov equation, we can show that the electron fluid is described by:

$$
\frac{\partial}{\partial t}\left(\frac{1}{2} n T\right)+\frac{\partial}{\partial x}\left(\frac{3}{2} n u T+Q\right)-u \frac{\partial}{\partial x}(n T)=0 .
$$

where $n(x, t)$ is the density, $T(x, t)$ is the effective temperature expressed in joules, $u(x, t)$ is the drift velocity and $Q(x, t)$ is the heat flux. We note that $T$ characterizes the non-drifting part of the electron velocity distribution, and it is not implied that this is Maxwellian. We assume that the positive ion density $n_{i}$ is defined by a suitable sheath model $[4,12]$, and that in this quasi-neutral region $n \simeq n_{i}$, so $n$ is given. From the specification of the current density, it follows that

$$
\begin{aligned}
\int_{0}^{s(t)} n d x & =n_{0}\left\{\frac{\tilde{u}_{l}}{\omega_{l}}\left(1-\cos \omega_{l} t\right)+\frac{\tilde{u}_{h}}{\omega_{h}}\left(1-\cos \omega_{h} t\right)\right\} \\
-(u n)_{0} & =n_{0}\left(\tilde{u}_{l} \sin \omega_{l} t+\tilde{u}_{h} \sin \omega_{h} t\right) .
\end{aligned}
$$

where $s(t)$ is the position of the electron sheath edge, with its origin at the ion sheath edge, $\tilde{u}_{l}=\tilde{J}_{l} / e n_{0}$, etc, and the subscript 0 refers to quantities defined at the ion sheath edge. 
We now assume that $T$ is independent of $x$ within the sheath, and with this assumption we can integrate eq. 1 between the ion sheath edge and the electron sheath edge to obtain the ordinary differential equation

$$
\begin{aligned}
\frac{1}{2} n_{0} \frac{d T}{d t} & \left\{\frac{\tilde{u}_{l}}{\omega_{l}}\left(1-\cos \omega_{l} t\right)-\frac{\tilde{u}_{h}}{\omega_{h}}\left(1-\cos \omega_{h} t\right)\right\} \\
& -T n_{0} \ln \left(\frac{n_{s}}{n_{0}}\right)\left(\tilde{u}_{l} \sin \omega_{l} t+\tilde{u}_{h} \sin \omega_{h} t\right)-2 Q_{b}\left(\frac{T}{T_{b}}\right)\left(1-\frac{T}{T_{b}}\right)=0,
\end{aligned}
$$

where $Q_{b}=\frac{1}{4} n_{0} \bar{v}_{b} T_{b}, \bar{v}_{b}=\sqrt{8 T / \pi m_{e}}$, the subscript $s$ denotes quantities defined at the electron sheath edge and $T_{b}$ is the temperature of the electron flux incident from the bulk plasma. This equation assumes that no electrons are absorbed by the electrode. Eq. 4 apparently contains six distinct physical parameters, namely $T_{b}, n_{0}, \tilde{J}_{l}, \omega_{l}, \tilde{J}_{h}$ and $\omega_{h}$. These, however, can be reduced to four dimensionless parameters, $\delta_{l} \equiv \tilde{u}_{l} / \bar{v}_{b}, \gamma_{l} \equiv \omega_{l} / \omega_{p}$, $\delta_{h} \equiv \tilde{u}_{h} / \bar{v}_{b}$ and $\gamma_{h} \equiv \omega_{h} / \omega_{p}$, where $\omega_{p}^{2} \equiv e^{2} n_{0} / \epsilon_{0} m_{e}$. We define $n_{s}(t)$ using the sheath model of [4], which has three control parameters. These are $\alpha \equiv \omega_{h} / \omega_{l}=\gamma_{h} / \gamma_{l}, \beta \equiv \tilde{J}_{h} / \tilde{J}_{l}=\delta_{h} / \delta_{l}$ and $H_{l} \equiv 8 \delta_{l}^{2} / \pi^{2} \gamma_{l}^{2}$. With the normalizations $\tau \equiv T / T_{b}$ and $\theta \equiv \omega_{l} t$, eq. 4 can be written:

$$
\begin{gathered}
\delta_{l}\left[(1-\cos \theta) \frac{d \tau}{d \theta}-2 \tau \ln \eta \sin \theta\right] \\
+\delta_{h}\left[\frac{1}{\alpha}(1-\cos \alpha \theta) \frac{d \tau}{d \theta}-2 \tau \ln \eta \sin \alpha \theta\right]+\tau(\tau-1)=0,
\end{gathered}
$$

where $\eta(\theta) \equiv n_{s} / n_{0}$.

Eq. 5 is solved by an expression in the form:

$$
\tau=\tau^{(0)}+\delta_{l} \tau_{l}^{(1)}+\delta_{h} \tau_{h}^{(1)}+\delta_{l}^{2} \tau_{l}^{(2)}+\delta_{h}^{2} \tau_{h}^{(2)}+\delta_{l} \delta_{h} \tau_{h l}^{(2)}+\mathrm{O}\left(\delta_{l, h}^{3}\right),
$$

where the $\tau^{(n)}$ are arbitrary functions that are to be determined by inserting the power series, eq. 6 , into the differential equation, eq. 5 . Since $\delta_{l, h} \ll 1$, the series may be truncated if $\alpha$ is not too large, and in this way, we find

$$
\begin{aligned}
\tau^{(0)} & =1 \\
\tau_{l}^{(1)} & =2 \ln \eta \sin \theta \\
\tau_{h}^{(1)} & =2 \ln \eta \sin \alpha \theta \\
\tau_{l}^{(2)} & =-(1-\cos \theta) \frac{d \tau_{l}^{(1)}}{d \theta} \\
\tau_{h}^{(2)} & =-\frac{1}{\alpha}(1-\cos \alpha \theta) \frac{d \tau_{h}^{(1)}}{d \theta} \\
\tau_{l h}^{(2)} & =-(1-\cos \theta) \frac{d \tau_{h}^{(1)}}{d \theta}-\frac{1}{\alpha}(1-\cos \alpha \theta) \frac{d \tau_{l}^{(1)}}{d \theta} .
\end{aligned}
$$


We can now express the sheath heating power per unit area as:

$$
\begin{aligned}
\left\langle S_{l h}\right\rangle & =-\left\langle Q_{0}\right\rangle=2 Q_{b} \tau(\tau-1) \\
& =2 Q_{b}\left\langle\delta_{l}^{2}\left(\tau_{l}^{(2)}+\tau_{l}^{(1)^{2}}\right)+\delta_{h}^{2}\left(\tau_{h}^{(2)}+\tau_{h}^{(1)^{2}}\right)\right\rangle \\
& =2 Q_{b}\left[\delta_{l}^{2} F_{0}\left(\alpha, \beta, H_{l}\right)+\delta_{h}^{2} F_{1}\left(\alpha, \beta, H_{l}\right)\right]
\end{aligned}
$$

as $\left\langle\tau_{l}^{(1)}\right\rangle=\left\langle\tau_{h}^{(1)}\right\rangle=\left\langle\tau_{l h}^{(2)}\right\rangle=0$, and where $F_{0}$ and $F_{1}$ are functions implied by the indicated time averaging. Scrutiny of these functions shows that their values depend only weakly on $\alpha$ and $\beta$, so that to a useful approximation $F_{0} \approx 36 H_{l} /\left(55+H_{l}\right)$, as in [6], and $F_{1} \approx 1.1 F_{0}$.

We will now discuss the predictions of this model with reference to particle-in-cell simulations [13]. Our procedure is described in detail in [14], therefore we give only brief particulars. We simulate only the sheath and its immediate vicinity, by injecting electrons and ions from one boundary with velocity distributions chosen to model an adjacent semiinfinite plasma with a given electron temperature and density. Electrons are injected with distribution corresponding to a Maxwellian bulk plasma, while ions are initially injected with a drift velocity at the Bohm speed and a thermal component at $350 \mathrm{~K}$. We allow this simulation to proceed in time until a harmonic steady state is reached with respect to both driving frequencies. Then, we freeze the ions, so that electrons cease to be collected by the electrode. In this way, we reproduce in the simulation the situation envisaged in the model discussed above, and we separate the collisionless heating effect from extraneous phenomena that would otherwise complicate our interpretation of the simulation results. The physical parameters used in these calculations are $n_{0}=5 \times 10^{15} \mathrm{~m}^{-3}$ and $T_{b}=30000 \mathrm{~K}$. Except where otherwise implied, $\omega_{l}=2 \pi \times 2 \mathrm{MHz}, \omega_{h}=2 \pi \times 26 \mathrm{MHz}, \tilde{J}_{l}=10 \mathrm{~A} \mathrm{~m}^{-2}$ and $\tilde{J}_{h}=36 \mathrm{~A} \mathrm{~m}^{-2}$ Indications of the general character of the heating effect, and the consistency of the simulations with the model, are shown in figs. 1 and 2. Fig 1 is an example of the heating power per unit area computed from the simulation. This figure shows that the heating power reaches a positive maximum during the expanding phase of the low-frequency sheath, where $\pi<\theta<2 \pi$, a feature which is also seen in the analytical model. In fig. 2, we compare the sheath electron temperature from the simulation with the analytical model, and we find generally good agreement. A more detailed parametric investigation is shown in fig. 3, which shows excellent agreement between the simulations and the model.

Our theory entails a surprising prediction: Namely, that the heating power may be greatly 
enhanced when two frequencies act together Specifically, using the expressions above,

$$
\frac{\left\langle S_{l h}\right\rangle}{\left\langle S_{l}\right\rangle+\left\langle S_{h}\right\rangle} \approx \frac{1+1.1 \beta^{2}}{1+\beta^{4} / \alpha^{2}}
$$

When $H_{l, h}$ are not too large so $I_{0} \sim 36 H_{l} / 55$, the maximum enhancement is approximately $(1+\alpha) / 2$, which occurs when $\beta \approx \sqrt{\alpha}$. These parameters are within the typical range of current experiments. In fig. 4, we compare simulations showing the heating effect produced by each frequency separately with their combined effect, demonstrating that the predicted enhancement does exist. In the case shown, which is close to the predicted maximum with $\beta / \sqrt{\alpha} \sim 1$, the enhancement factor is approximately 5 , compared to the predicted 7 . The discrepancy is mostly because $H_{h}$ is significantly less than 1 , and the single frequency theory is not highly accurate in that case. As fig. 4 suggests, the mechanism of the enhancement is the modification of the sheath structure that is produced by applying the lower frequency current. Evidently, the sheath volume and the density gradients within it are increased appreciably in the presence of the lower frequency current. In the present theory, the average heating power is exactly zero on the plasma side of the ion sheath edge, because both $n$ and $T$ are assumed to be uniform in that region. In simulation, and presumably in experiments, there is a region of negative heating in the bulk plasma adjacent to the sheath, which is clearly visible in fig. 4. This effect has been discussed elsewhere [7, 10, 11]. In fig. 5 we compare the theory discussed above with simulations, for the case where $J_{h}$ is held constant and $J_{l}$ is varied. There is good agreement, except in the limit $J_{l} \rightarrow 0$, where our theory cannot be applied-because the sheath model of [4] is not valid. However, by an ansätz we can construct an expression that gives a practically useful result for a wide range of parameters: We replace $H_{l}$ in eq. 15 with an effective value $H_{\text {eff }}=\sqrt{H_{l}^{2}+H_{h}^{2}}$. As shown in fig. 5 , this gives a result in good agreement with simulation, even when $\beta / \alpha \gg 1$.

In summary, we have developed an analytical model for collisionless electron heating in a dual frequency discharge that quantitatively predicts the main features of the heating effect as it is seen in kinetic simulations and in the only relevant experiments yet available. The model predicts that the collisionless heating effect is greatly enhanced in the presence of two frequencies acting together, because both the affected volume of plasma and the dynamic range of density are increased by applying the low frequency. This is likely to be an effect of considerable practical importance. The model can also be reduced to a convenient analytical formula, eq. 15, that reproduces the area integrated heating power 
with excellent accuracy for typical values of $\alpha$ and $\beta$, and provides a reasonable estimate for more extreme parameters. The present theory begins to fail when $\alpha \gg 1$, when it is in probable that electromagnetic effects should be considered $[15,16]$, and that one no longer has anything resembling a traditional capacitive discharge. Nothing is yet known of the electron heating processes that might prevail under such conditions.

* Electronic address: miles.turner@dcu.ie

[1] V. A. Godyak and R. B. Piejak, Phys. Rev. Lett. 65, 996 (1990).

[2] O. A. Popov and V. A. Godyak, J. Appl. Phys. 57, 53 (1985).

[3] H. C. Kim and J. K. Lee, Phys. Rev. Lett. 93, 085003 (2004).

[4] J. Robiche, P. C. Boyle, M. M. Turner, and A. R. Ellingboe, J. Phys. D: Appl. Phys. 36, 1810 (2003).

[5] H. C. Kim, J. K. Lee, and J. W. Shon, Phys. Plasmas 10, 4545 (2003).

[6] G. Gozadinos, M. M. Turner, and D. Vender, Phys. Rev. Lett. 87, 135004 (2001).

[7] M. M. Turner, Phys. Rev. Lett. 75, 1312 (1995).

[8] V. A. Godyak, Sov. Phys.-Tech. Phys. 16, 1073 (1972).

[9] M. A. Lieberman, IEEE Trans. Plasma Sci. 16, 638 (1988).

[10] M. Surendra and D. B. Graves, Phys. Rev. Lett. 66, 1469 (1991).

[11] I. D. Kaganovich, Phys. Rev. Lett. 89, 265006 (2002).

[12] K. Denpoh, G. Wakayama, and K. Nanbu, Jpn. J. Appl. Phys. 43, 5533 (2004).

[13] C. K. Birdsall and A. B. Langdon, Plasma Physics via Computer Simulation (McGraw-Hill, New York, 1985).

[14] G. Gozadinos, D. Vender, and M. M. Turner, J. Comp. Phys. 172, 348 (2001).

[15] M. A. Lieberman, J.-P. Booth, P. Chabert, J.-M. Rax, and M. M. Turner, Plasma Sources Sci. Technol. 11, 283 (2002).

[16] P. Chabert, J. L. Raimbault, P. Levif, J. M. Rax, and M. A. Lieberman, Phys. Rev. Lett. 95, Art. No. 205001 (2005). 


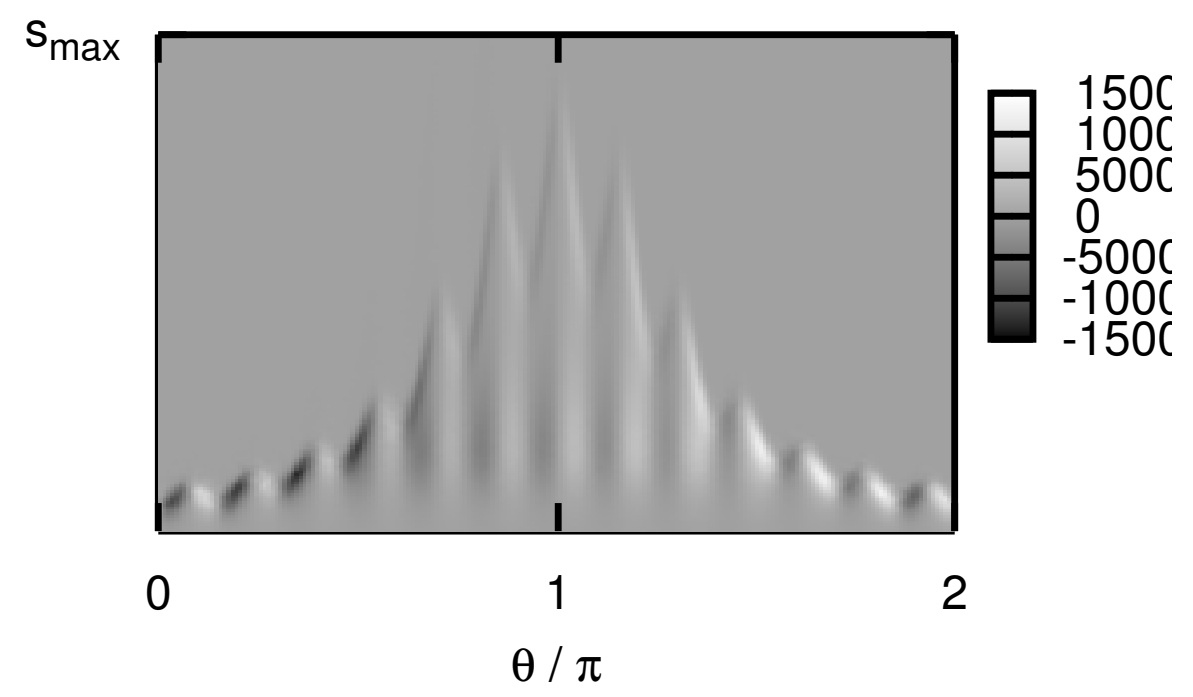

FIG. 1: Collisionless heating power as a function of space and time, calculated by the particle simulation, with $\tilde{J}_{l}=10 \mathrm{~A} \mathrm{~m}^{-2}, \omega_{l}=2 \pi \times 2 \mathrm{MHz}, \tilde{J}_{h}=20 \mathrm{~A} \mathrm{~m}^{-2}$ and $\omega_{l}=2 \pi \times 26 \mathrm{MHz}$, and the plasma parameters discussed in the text. The ion sheath edge is at $x=0$ and the electrode is at $x=s_{\max }$. 


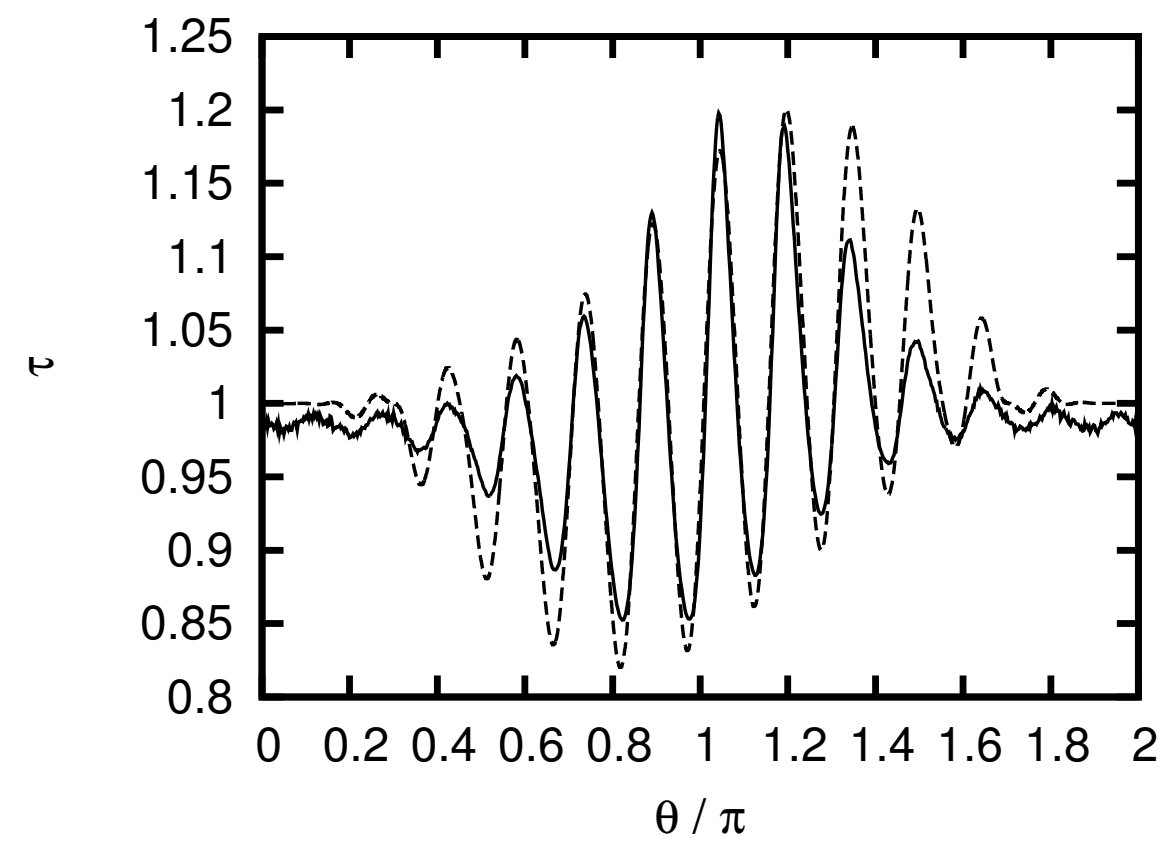

FIG. 2: Temperature of electrons in the sheath computed from the analytic theory of the text (solid line) and from the particle-in-cell simulation (dashed line), for the conditions noted in fig. 1.

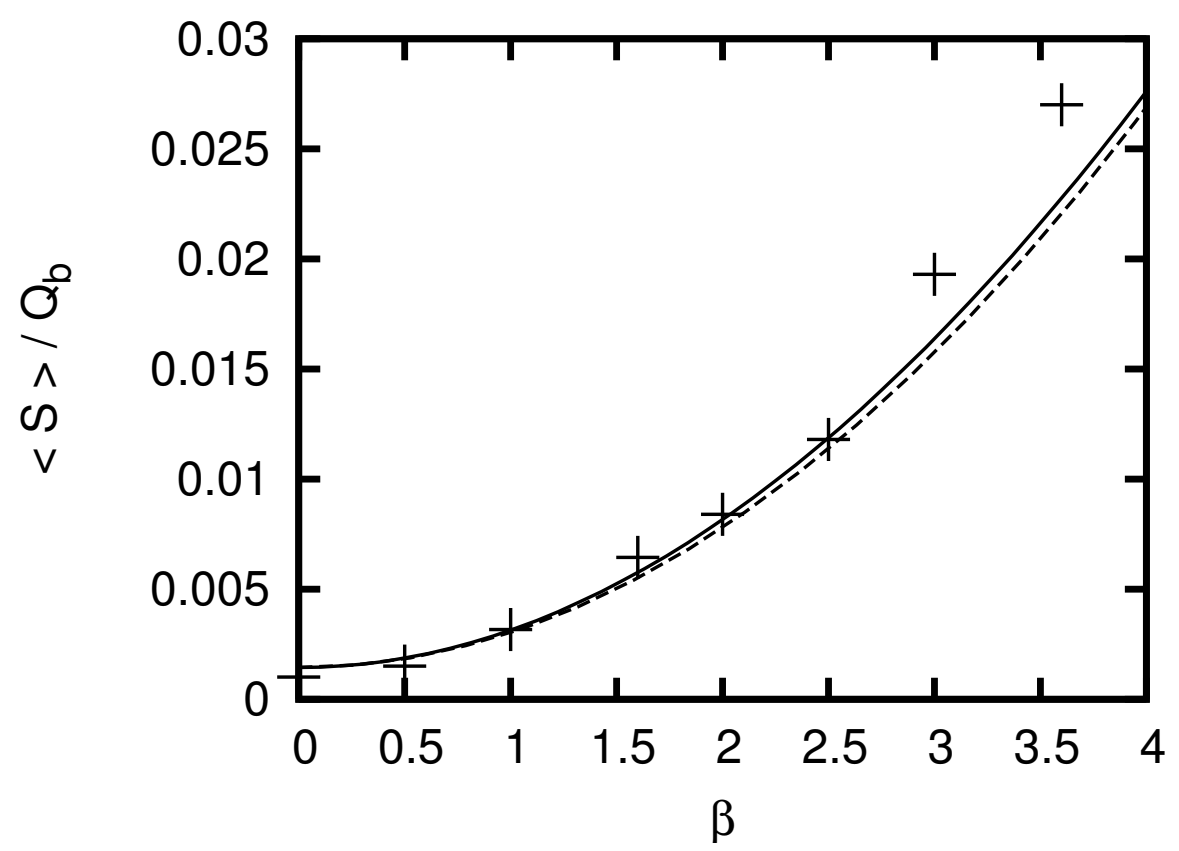

FIG. 3: Normalized collisionless heating power as a function of $\beta \equiv \tilde{J}_{h} / \tilde{J}_{l}$, with $\tilde{J}_{h}$ as the parameter varied (so that $H_{l}$ is constant) and with $\alpha=\omega_{h} / \omega_{l}=13$. 


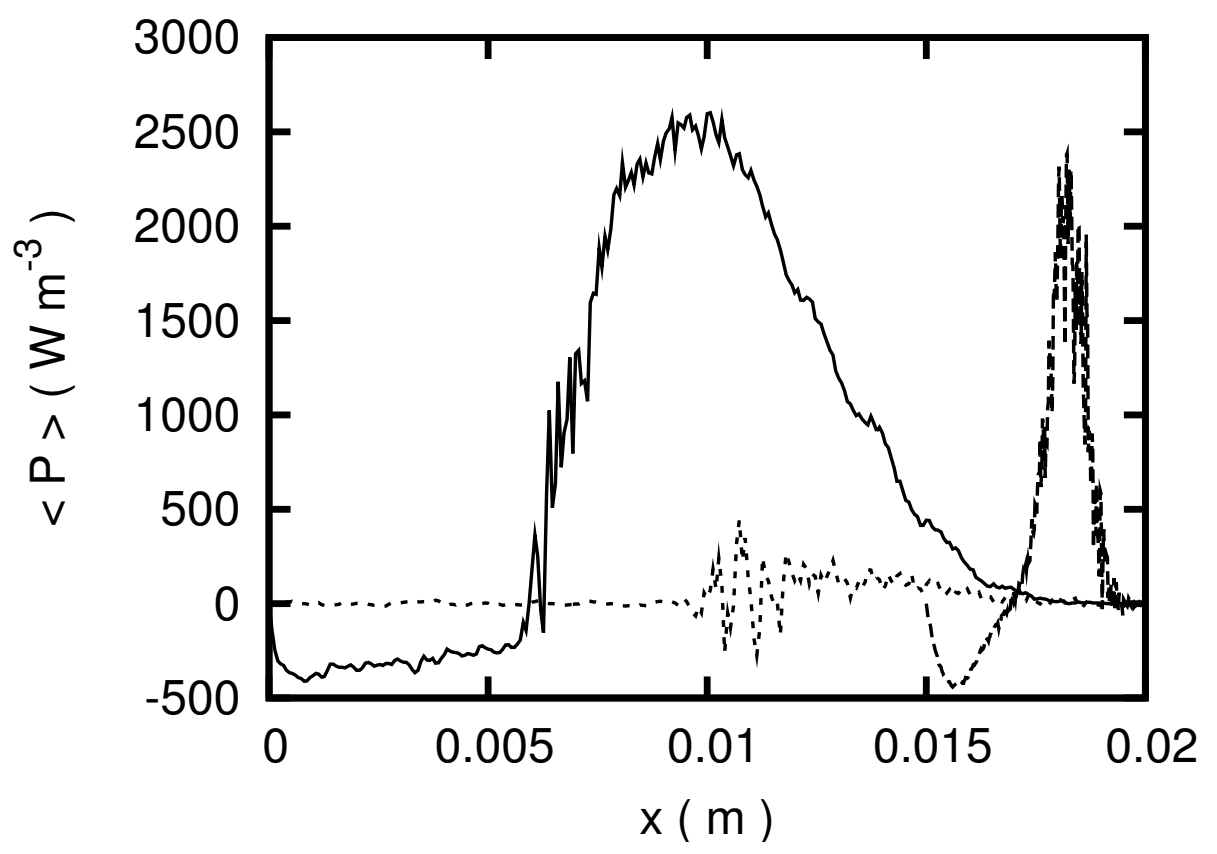

FIG. 4: Time averaged electron heating power resolved in space for three cases corresponding to the two frequencies acting separately and both acting together. Note that the ion sheath edge in each case is approximately located at the transition to negative power absorption, and that the sheath width is appreciably influenced by the application of the high frequency [4], relative to the low frequency acting alone. The parameter values used are $\tilde{J}_{l}=10 \mathrm{~A} \mathrm{~m}^{-2}, \omega_{l}=2 \pi \times 2 \mathrm{MHz}$, $\tilde{J}_{h}=36 \mathrm{~A} \mathrm{~m}^{-2}$ and $\omega_{l}=2 \pi \times 26 \mathrm{MHz}$. 


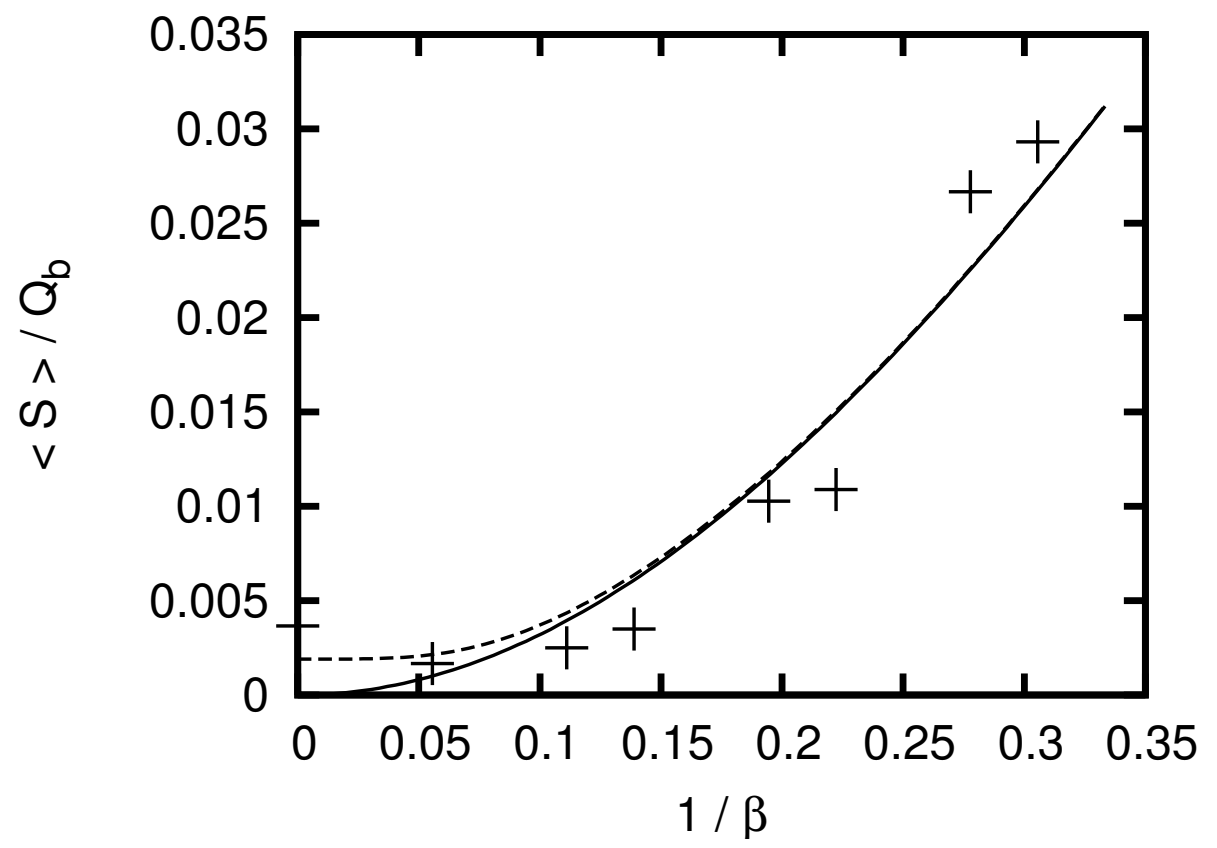

FIG. 5: Time averaged electron heating power as a function of $1 / \beta \equiv J_{l} / J_{h}$, where the parameter varied is the low frequency current density $J_{l}$. Other parameters held constant are the high frequency current density $J_{h}=36 \mathrm{~A} \mathrm{~m}^{-2}$, and the two frequencies $\omega_{l}=2 \pi \times 2 \mathrm{MHz}$ and $\omega_{h}=$ $2 \pi \times 26 \mathrm{MHz}$. The points are simulation data. The short dashed line is the model of eq. 15 , the long dashed line the model generalized using the ansätze of the text. 